\section{Mixed reactions}

\section{Malcolm Scott}

Nuclear Physics for Engineers and Scientists: Low Energy Theory with Applications Including Reactors and Their Environmental Impact. By S. E. Hunt. Ellis Horwood: 1987. Pp.371. £51.50, \$116.40. Principles of Nuclear Science and Engineering. By A. A. Harms. Research Studies Press, Letchworth, Herts/Wiley, New York: 1987. Pp.192. £22, \$43.05.

Although the basic aim of these two books is similar, namely provision of the physics background necessary to understand fission and fusion reactors, the approaches and achievements could not be more disparate. Many scientific books claim to be written simply, with little or no assumed mathematics, whereupon the author starts by solving a second-order differential equation. Professor Hunt states in his short introduction that he wishes to emphasize the physical interpretation of nuclear behaviour, in order to make the subject intelligible to a nonphysicist - and he proceeds to do just that. Indeed, the text has an elegance, yet simplicity, of presentation which one must admire and would have to strive very hard to emulate. Not only does Hunt explain the underlying principles clearly, but he manages to do so in a very readable way, introducing small personal touches which help to take away some of the impersonality associated with science (". . . many man years, including some of the author's own, were expended on ...").

The book starts with a chapter on the nuclear atom, and the next six are devoted to basic nuclear and atomic physics. There follow five chapters dealing with the production, properties and detection of charged particles, photons and neutrons. The physics of fission and fusion is covered in two separate chapters, while the concluding three deal with commercial fission reactors, fuel reserves (nuclear and non-nuclear) and nuclear safety.

In a book of this breadth, an author cannot have had first-hand professional involvement in every topic discussed. There are, however, only a few instances where this has led to statements which are slightly questionable, or where the emphasis gives a misleading impression. There are, surprisingly, a number of typographical errors, and a whole chapter has the wrong running headline. Most of the slips are easily spotted, but if the mean current of the Nuffield Cyclotron were really $500 \mathrm{MA}$ we would be running a power station not a research accelerator!

Professor Hunt's book is valuable for several different classes of reader. It could serve both as refresher course for those who have already studied the field, or as an introduction for those who have not. Its breadth and lucidity are impressive, and it achieves its depth of understanding without resorting to recipes. Only the cost of the hardbound edition is off-putting. One can only hope that a paperback version will soon be forthcoming, so that the book can reach the readership it deserves.

In contrast, Professor Harms's book starts with a relatively long preface in which he states his pedagogical intention of applying a "bottom-up" approach to nuclear fission and fusion, contrasted with a "top-down" approach, involving "simplification of a complex and specialised subject by a variety of conceptual reductions and analytical approximations".

With such a claim, I expected a text either having simple rigour or offering insights disguised by alternative approaches. Unfortunately, this is not the case. One reason is simply the prose style, which is often long-winded and ponderous. Another is that, unlike in many modern textbooks, areas which may be conceptually difficult have not been presented with the potential problems

\section{Odd, isn't it?}

\section{Tony Sudbery}

From Paradox To Reality: Our Basic Concepts of the Physical World. By Fritz Rohrlich. Cambridge University Press: 1987. Pp.227. £25, \$34.50.

Particles and Paradoxes: The Limits of Quantum Logic. By Peter Gibbins. Cambridge University Press: 1987. Pp.181. $H b k £ 25, \$ 34.50 ; p b k £ 8.95, \$ 11.95$.

SINCE the early decades of this century, the popular image of theoretical physics has been surrounded by an aura of paradox. The two revolutionary theories of that period, relativity and quantum mechanics, seemed to violate not only common sense but all rationality. In consequence the basic concepts of theoretical physics are an appropriate subject of serious study for more than just physics students, and these two books offer reading matter for different parts of this wider audience.

Fritz Rohrlich's book is based on an undergraduate course "Concepts in Contemporary Physics" that he offers to students majoring in non-science subjects. As well as teaching them about the specific concepts of relativity and quantum mechanics, he is concerned to give a general understanding of the way scientific theories fit together and what happens when a theory is superseded in a scientific revolution. Rohrlich's view is that this is bound to happen to every theory, and that only when it does is the theory complete, or 'established', because only then does it acquire a definition of its domain of valid- faced by the reader clearly in mind. For example, in discussing the question of fission reactor criticality the same symbol is used for materials and geometric buckling, thus obscuring the distinction between them, a confusion only partly resolved by the text. Only in one of the problems at the end of the chapter is the origin of this distinction hinted at, and the reader is left to puzzle things out. Similarly, the reason for the fundamental mode describing the spatial distribution of the neutron flux in a critical assembly is only alluded to, yet giving the timedependent solution would both make the reason clear and, I would have thought, represent a true "bottom-up" approach.

Unfortunately, one can cite many instances where simple points are either obscured or are laboured. Given the range of the textbooks which already cover this field, it is, sadly, difficult to recommend this one.

Malcom Scott is a Senior Lecturer in the Department of Physics, University of Birmingham, $P O$ Box 363, Birmingham B15 2TT, UK.

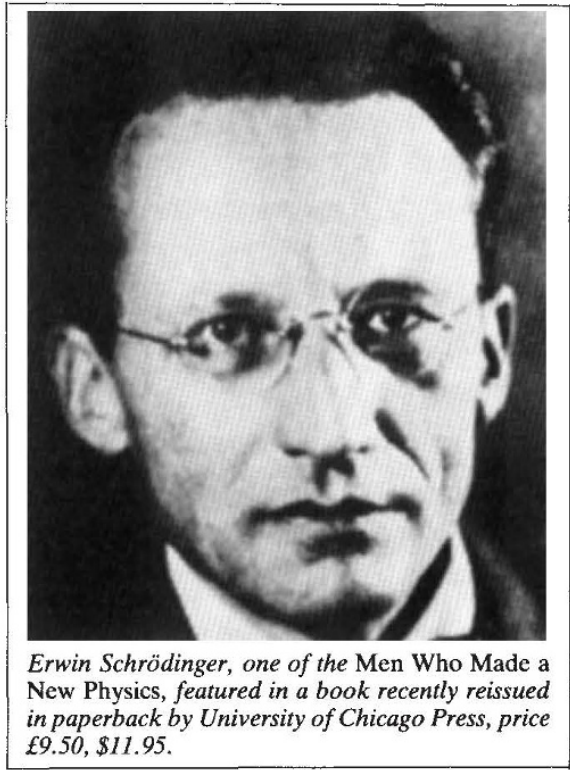

ity. This is a clear and coherent account of a doctrine which is often impressed on science students, though I hope some of Rohrlich's readers will wonder if it really is the necessary truth he says it is.

In his accounts of special relativity and quantum mechanics, Rohrlich focuses on the central concepts. He places both theories in their historical context, describing their roots in earlier ideas and the subsequent delimitation of their validity by general relativity and quantum field theory, respectively. The author does not assume any knowledge of physics, but his readers are expected to work (and they may be puzzled by the physicist's use of such words as 'interfere' and 'scatter').

The paradoxical aspects of the concepts are confronted and, in the case of special 